\title{
Hybrid models for hardware-in-the-loop simulation of hydraulic systems Part 1: theory
}

\author{
J A Ferreira ${ }^{1 *}$, F Gomes Almeida ${ }^{2}$, M R Quintas ${ }^{2}$ and $\mathbf{J}$ P Estima de Oliveira $^{3}$ \\ ${ }^{1}$ Department of Mechanical Engineering, University of Aveiro, Aveiro, Portugal \\ ${ }^{2}$ IDMEC-Polo FEUP, University of Porto, Porto, Portugal \\ ${ }^{3}$ Department of Electronic Engineering, University of Aveiro/IEETA, Aveiro, Portugal
}

\begin{abstract}
Physical modelling of hydraulic systems results in systems of differential and algebraic equations (DAEs) that are normally stiff. This is because they often include multiple temporal scales and present complex and non-linear behaviour. The simulation of these stiff DAEs in real time demand small time steps, in order to achieve algorithm stability, because explicit fixed step solvers are usually applied. Modelling some fast dynamics as instantaneous changes in order to reduce the model's stiffness has been an area of research in the last few years. The description of the dynamic behaviour as piecewise continuous modes, interspersed with discrete transitions, together with the reduction in the model complexity, facilitates the use of fixed time step integration methods and thus allows real-time simulation.

The main goal of this work was to obtain not too complex models in order to allow the models to be used in hardware-in-the-loop experiments. This paper proposes the use of the statecharts formalism to describe hybrid behaviour in hydraulic systems and presents semiempirical models of a valve controlled hydraulic cylinder intended to test real controller performance on a hardware-in-the-loop setup. These semiempirical models require less computing power, and it is easier to adjust the model parameters.
\end{abstract}

Keywords: fluid power, modelling, real-time simulation, hardware-in-the-loop simulation

NOTATION
$a_{\mathrm{p}}$
$A_{\mathrm{s} 1}, A_{\mathrm{s} 2} A_{1 \mathrm{t}}, A_{2 \mathrm{t}}$
$A_{1}, A_{2}$
$f_{n}, \omega_{n}$
$F_{\mathrm{CO}}$
$F_{\mathrm{f}}$
$F_{\mathrm{L}}$
$F_{\mathrm{S}}$
$g$
$g_{1 \mathrm{kc}}$
$k_{1}, k_{2}, k_{3}, k_{4}, k_{5}$
$k_{1 \mathrm{t}}, k_{2 \mathrm{t}}, k_{3 \mathrm{t}}, k_{4 \mathrm{t}}, k_{5 \mathrm{t}}$
$K_{\mathrm{v}}$

piston acceleration

pseudo-sections

cylinder chamber areas

natural (angular) frequency

Coulomb frictional force

frictional force

load applied force

Stribeck friction

acceleration due to gravity

cylinder leakage conductance

pseudo-section parameters

pseudo-section parameters

viscous friction coefficient

The MS was received on 20 February 2004 and was accepted after revision for publication on 25 May 2004.

* Corresponding author: Department of Mechanical Engineering, University of Aveiro, Campo Universitario, Aveiro 3810, Portugal. E-mail: jaff@mec.ua.pt

$\bar{K}_{p 0}$
$\bar{K}_{q 0}$
$L_{v}, L_{a}$
$M$
$P_{i}$
$P_{\mathrm{L}}$
$P_{\mathrm{n}}$
$P_{1}, P_{2}$
$\bar{P}_{\mathrm{L}}$
$q_{i j}$

$q_{1 \mathrm{k}}$

$q_{1 \mathrm{kc}}$

$Q_{\text {in }}, Q_{\text {out }}$ relative pressure gain at $\bar{x}_{\mathrm{s}}=0$

flow gain at $\bar{x}_{\mathrm{s}}=0$

cylinder maximum stroke spool velocity and acceleration limits respectively connected mass (load, piston and rod)

relative pressure at valve port $i$ load pressure drop nominal pressure drop cylinder chamber relative pressures relative load pressure volumetric flowrate from port $i$ to port $j$ leakage volumetric flowrate cylinder leakage volumetric flowrate incoming and outgoing volumetric flowrates respectively 


\begin{tabular}{|c|c|}
\hline $\begin{array}{l}Q_{\mathrm{L}} \\
Q_{\mathrm{n}} \\
Q_{\mathrm{s}}, Q_{\mathrm{t}}\end{array}$ & $\begin{array}{l}\text { load volumetric flowrate } \\
\text { nominal volumetric flowrate } \\
\text { tank and source volumetric } \\
\text { flowrates resnectively }\end{array}$ \\
\hline$Q_{1}, Q_{2}$ & $\begin{array}{l}\text { volumetric flowrates of outlet } \\
\text { ports }\end{array}$ \\
\hline$T$ & temperature \\
\hline $\bar{u}$ & $\begin{array}{l}\text { normalized valve input } \\
\in[-1,1]\end{array}$ \\
\hline$v_{\mathrm{p}}$ & piston velocity \\
\hline & Stribeck velocity \\
\hline$V_{\mathrm{L} 1}, V_{\mathrm{L} 2}$ & $\begin{array}{l}\text { enclosed volumes at lines } 1 \text { and } \\
2 \text { respectively }\end{array}$ \\
\hline$W_{\text {in }}, W_{\text {out }}$ & $\begin{array}{l}\text { incoming and outgoing weight } \\
\text { flowrates respectively }\end{array}$ \\
\hline$x_{\mathrm{p}}$ & piston position \\
\hline $\bar{x}_{\mathrm{s}}$ & $\begin{array}{l}\text { normalized valve spool position } \\
\in[-1,1]\end{array}$ \\
\hline$z$ & $\begin{array}{l}\text { seal deformation (friction } \\
\text { model) }\end{array}$ \\
\hline$\beta$ & oil bulk modulus \\
\hline$\beta_{\mathrm{e} 1}$ & $\begin{array}{l}\text { effective bulk modulus for } \\
\text { chamber } 1\end{array}$ \\
\hline$\beta_{\mathrm{e} 2}$ & $\begin{array}{l}\text { effective bulk modulus for } \\
\text { chamber } 2\end{array}$ \\
\hline$\Delta P_{i j}$ & $\begin{array}{l}\text { pressure drop between port } i \\
\text { and port } j\end{array}$ \\
\hline$\Delta P_{m}$ & $\begin{array}{l}\text { pressure difference to the middle } \\
\text { point }\end{array}$ \\
\hline$\xi$ & damping ratio \\
\hline$\rho$ & mass density \\
\hline$\sigma_{0}$ & seal stiffness (friction model) \\
\hline$\sigma_{1}$ & $\begin{array}{l}\text { seal damping coefficient } \\
\text { (friction model) }\end{array}$ \\
\hline
\end{tabular}

\section{INTRODUCTION}

Hydraulic systems have been used, for a long time, in industrial manufacturing and in heavy machinery. Hydraulic hardware has undergone great evolution during the last few years, from hydromechanical devices to sophisticated electrohydraulic systems controlled by microprocessors. The use of electronics and microprocessors contributes to improving the dynamic performance and to adding new features to traditional systems. Hydraulic systems usually have complex dynamics and non-linearities that lead to hard tasks, even danger, the parametrization of controllers and the testing of new control algorithms. The use of a new control schemes, usually applied to non-linear systems, such as adaptive control, fuzzy logic or neural networks, to name just a few, becomes possible due to the introduction of microprocessors in the control cycle and to the technological evolution of hydraulic hardware. Also, with the evolution of the computing power, more complex systems can be simulated in real time with decreasing costs. All these factors made possible the use of hardware-inthe-loop simulation (HILS) techniques [1], until recent years almost proprietary to the aerospace industries, with respect to other engineering fields. HILS refers to a technology in which some of the components of a pure simulation are replaced with actual hardware. This type of procedure is useful, for example, to test a controller, which, instead of being connected to the real equipment under control, is connected to a real-time simulator.

Hydraulic systems normally present complex and non-linear dynamic behaviour and often include multiple temporal scales. Modelling these systems results in nonlinear equation systems that are usually stiff. When using numerical simulation, the integration algorithms change their time step to handle these multi-time scale behaviours. However, these variable-step methods are unsuitable for real-time simulation applications. For realtime simulation, a fixed-step integration algorithms must be used. The fixed-step methods mainly use explicit algorithms that generally have problems when solving stiff systems. In fact, when some variables in the system undergo fast changes, and the step size is too large, fixedstep algorithms may become unstable or the solution may diverge.

Reduction in the overall model stiffness can be achieved by modelling some fast dynamics as instantaneous changes and representing the dynamic behaviour as piecewise continuous modes interspersed with discrete transitions [2]. These different continuous modes of operation can be described with expressive formalisms such as the hybrid bond graphs [3], hybrid automata [4] or hybrid Petri nets [5]. However, these are flat formalisms and so complex hybrid dynamics are difficult to describe and understand. Another formalism such as statecharts [6] added to hybrid features $[\mathbf{7}, \mathbf{8}]$ is more suitable to describe complex dynamics due to its hierarchical approach to express behaviour. The capability of clearly describing, in a structured way, complex hybrid behaviours helps the specialist modeller in his or her task. It enables an easier evaluation of the impact and tradeoffs involved when fast continuous behaviours are substituted by discrete transitions. Other additional problem, when modelling for real-time simulation, is the model complexity that, associated with limited step sizes, implies high performance computing hardware in order to guarantee step size restrictions. One way to reduce the computing power and consequently running the real-time simulation in lowcost hardware is to decrease the model complexity. This can be achieved by using semiempirical models for the components instead of using pure physical models. These semiempirical models should capture the most important characteristics of the system behaviour for control and simulation purposes. Semiempirical models may be used when a model of the input/output component behaviour is sufficient for simulation purposes. This is the usual case when simulations are used for system design. If an 
internal component behaviour description is needed, as in the case of component design, then physical models must, in general, be used, compromising its real-time capabilities.

These two complementary approaches, multi-mode continuous behaviour and semiempirical models, facilitate the use of fixed-time step integration methods and thus allow real-time simulation of stiff systems.

\section{BEHAVIOUR DESCRIPTION WITH THE STATECHARTS FORMALISM}

To overcome the above limitations of finite state machine (FSM) formalism, Harel [6] proposed statecharts as a visual formalism for the specification and modelling of complex reactive systems. The graphical syntax for statecharts has been developed quite early; however, the definition of formal semantics is not yet established. In accordance with the types of system to be modelled, several variants of statecharts have been proposed [9]. The main enhancements introduced by the statecharts formalism on the FSM were the use of hierarchy, parallelism and broadcast communication.

Hierarchy is a well-accepted approach for designing and managing complex designs. In statecharts, hierarchy is used to group sets of states together, allowing highlevel description and stepwise development. Depth is achieved by an OR decomposition of a conventional FSM state, while orthogonality is introduced by an AND decomposition. Parallelism inside a statechart is described by AND states, allowing modelling of concurrent activities in the same model through orthogonal states. These orthogonal states are all activated when an AND state is entered and are all deactivated when it is exited.

Figure 1 shows the high-level description of a system with two states $\mathrm{A}$ and $\mathrm{B}$. These states can be refined through state decomposition. State A will be a compound OR state with substates A1, A2 and A3. State B will

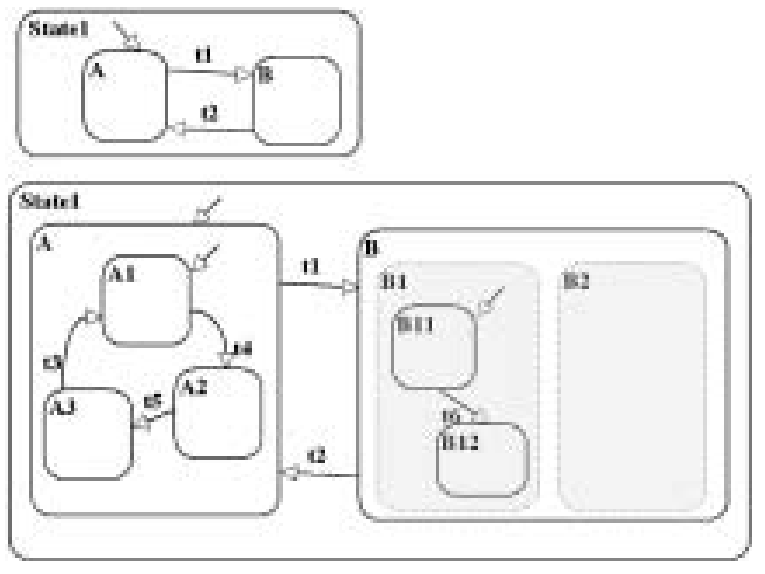

Fig. 1 Refining states by AND/OR decomposition

I03404 (C) IMechE 2004 be a compound AND state with substates B1 and B2. This process can proceed until low-level description is achieved. When orthogonal components are not truly independent, communication between them is specified by associating an action with a transition. This action is assumed to be broadcasted and so every system component in the statechart will recognize the message. This broadcast communication mechanism allows, when one part generates an event, that all the other parts sense it, acting in response if it is so specified.

Other enhancement over the FSM formalism is the association of an event action with a transition, when it is fired, or with a state, while the state is entered or exited. Continuous activity, usually expressed with differential and algebraic equations (DAEs), can also be associated with a state for modelling continuous behaviour when the state is active. This action and activity concepts allow the modelling of hybrid systems; actions capture the discrete systems' features while activities describe the continuous part. Initialization of the state variables of DAEs can be carried out by associating event actions with transitions or with the entrance or exiting of states. This variant of statecharts is usually called hybrid statecharts and can be viewed as DAEs with its variable structure controlled by the discrete statechart features. Changing the DAE structure corresponds to substituting a set of continuous equations by another. This is described in a hybrid statechart by the continuous equations (expressed with the continuous activity) that belong to different states. However, changing the equations in a DAEs can vary the index of the DAEs which can cause problems to numerical solvers.

Consider the example of a relief valve that limits the pressure in a hydraulic system. It is a hydromechanical closed-loop system but it is usually modelled by its (static) input/output characteristic [10]. The behaviour of the simple relief valve model can be described by considering that the valve has two possible stable states, and also hysteresis when it changes from one state to another. The valve volumetric flowrate $q_{\mathrm{r}}$ through the valve orifice is assumed laminar and is represented by two conductances, one that is the leakage conductance when the valve is closed, $g_{1 \mathrm{k}}$, and the other the conductance when the valve is opened, $g_{\text {op }}$. The interpretation of the relief valve statechart (Fig. 2) can be made in the following way: when the pressure difference $\Delta P$ is greater than the pressure required to open the valve, $p_{\text {Open }}$, the valve will be opened $\left(q_{\mathrm{r}}=\left(\Delta P-p_{\text {Close }}\right) g_{\text {op }}+\Delta P g_{1 \mathrm{k}}\right)$; when the pressure difference is smaller than the closed pressure $p_{\text {Close }}$ the valve will be closed $\left(q_{\mathrm{r}}=\Delta P g_{\mathrm{lk}}\right)$.

\section{MODELLING A HYDRAULIC SYSTEM}

This section presents semiempirical and hybrid models for some hydraulic components and describes how the statecharts formalism can be used to express the system's

Proc. Instn Mech. Engrs Vol. 218 Part I: J. Systems and Control Engineering 


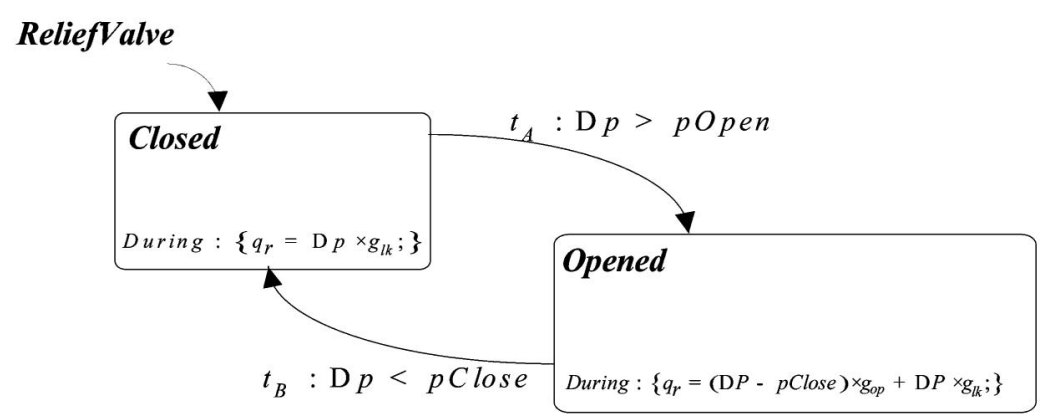

Fig. 2 Behaviour description of a relief valve using statechart

hybrid behaviour. The experimental system used for modelling purposes consists of a hydraulic cylinder driven by a high-performance proportional valve. The two complementary modelling approaches are used to model the proportional valve (semiempirical model) and to model the hydraulic cylinder (hybrid statechart).

The double-action cylinder has low-friction seals in order to minimize the stick-slip phenomena. The cylinder load is a mass $M$ in the vertical position. Figure 3 presents the schematic diagram of the valve spool and the cylinder.

\subsection{Valve model}

The valve model is split into two main blocks with a serial connection. The first block represents the dynamic part where the electrical signal of the controller card is the input and the spool position the output. It describes the different spool dynamics for different input amplitudes applied to the valve. The second block deals with the static behaviour and relates the volumetric flowrate through the valve with the input spool position and port pressures.

\subsubsection{Spool motion}

The amplitude and phase frequency response are the basis for the development of the model to describe the valve spool motion. The hysteresis for this type of valve is less then 0.5 per cent and the other disturbances such as spool friction or flow forces are minimized due to the closed-loop control of the spool position through an electronic control card. The most important behaviour to be modelled is the frequency response of the spool position with regard to the input signal, as the main use of the present model is for control experiments.

The main objective is to reproduce the frequency response amplitudes with a second-order model with acceleration and velocity saturation. The phase lag is adjusted with a delay as in reference [11].

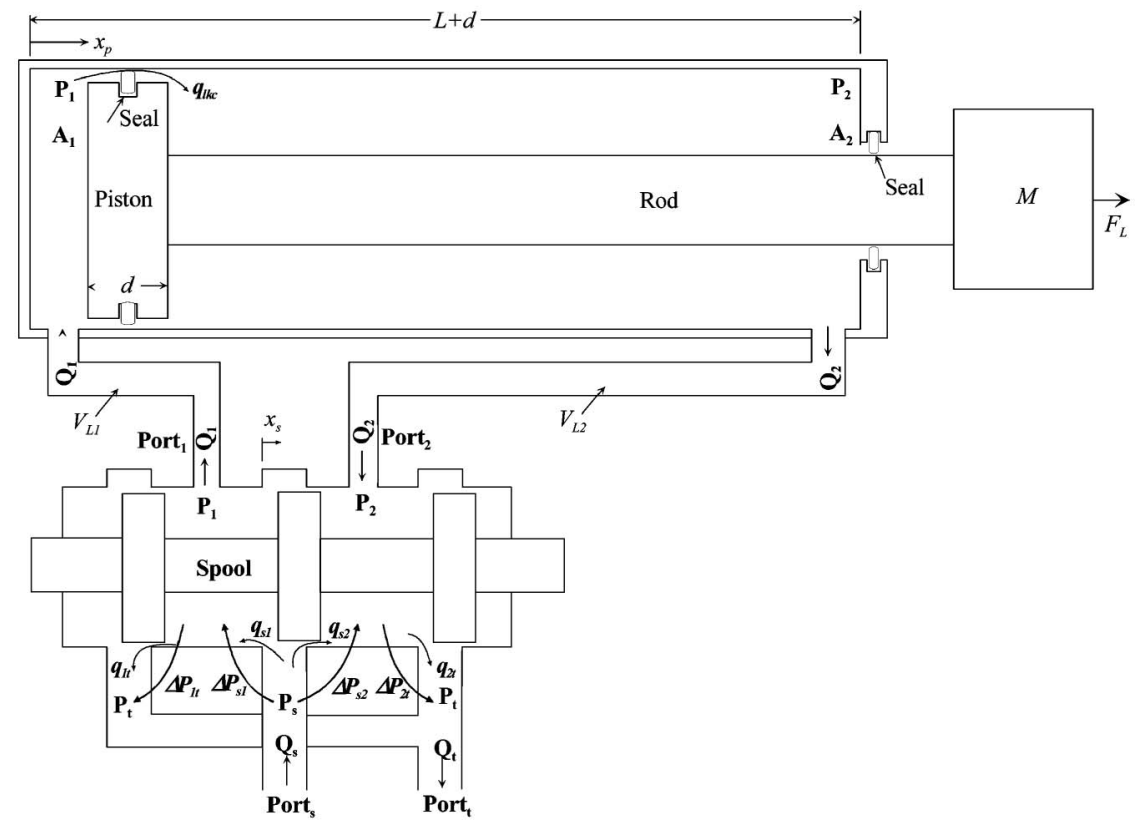

Fig. 3 Schematic diagram of the cylinder and valve 


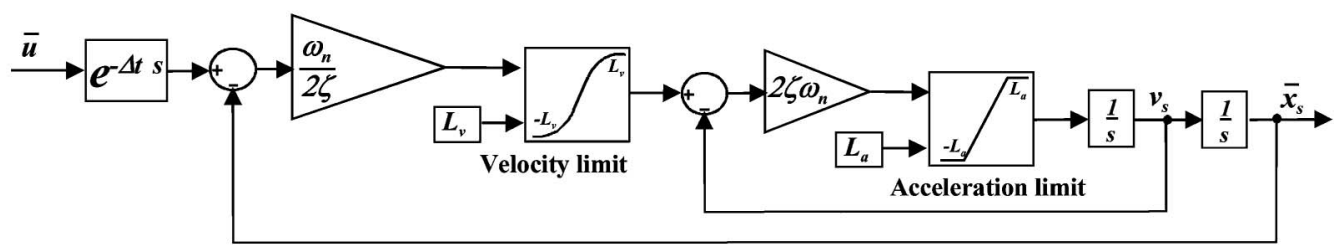

Fig. 4 Dynamic model for spool position with velocity and acceleration limits

Several simulations were made for different model complexities: with velocity saturation only; with velocity and acceleration saturation. Different non-linear limiter shapes, based on continuous and variable structure functions, were considered in the model presented in Fig. 4. The best results were obtained with a limiter based on the arctangent function for the velocity and a saturation limiter for the acceleration [12]. It should be noted that both limiters have a unitary derivative at the origin.

\subsubsection{Volumetric flowrate model}

The spool position controls the volumetric flowrate into the cylinder chambers with a non-linear function of the normalized spool position $\bar{x}_{\mathrm{s}}$ and the pressure difference $\Delta P$. This flow, combined with leakage and compressible flows, controls the chamber pressures. Figure 5 presents a Wheatstone bridge representing the valve flowrates, pressures and orifice sections.

The valve orifice areas versus spool position $\bar{x}_{\mathrm{s}}$ are modelled with pseudo-section functions $A_{i j}\left(\bar{x}_{\mathrm{s}}\right)$ as in reference [11]. The volumetric flowrate is assumed to be always turbulent, the laminar flow being implicitly modelled by the pseudo-section curves, $A_{i j}\left(\bar{x}_{\mathrm{s}}\right)$. The volumetric flowrate $q_{i j}$ through a restriction section from port $i$ to port $j$ is written as $[\mathbf{1 3}, \mathbf{1 4}]$

$$
q_{i j}=\operatorname{sgn}\left(\Delta P_{i j}\right) A_{i j}\left(\bar{x}_{\mathrm{s}}\right) \sqrt{\left|\Delta P_{i j}\right|}
$$

where $\Delta P_{i j}=P_{i}-P_{j}$ is the pressure drop between the two ports and $\operatorname{sgn}\left(\Delta P_{i j}\right)$ is the sign of $P_{i} \in P_{j}$. Considering the nomenclature presented in Figs 3 and 5 , the valve

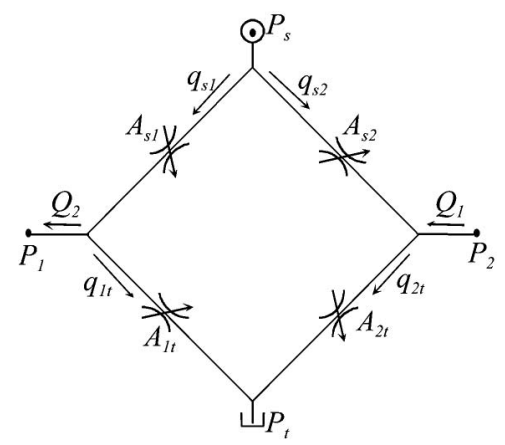

Fig. 5 Wheatstone bridge representation of valve flowrates, pressures and orifice pseudo-sections

I03404 C IMechE 2004 flow equations are given by

$$
\begin{aligned}
& q_{\mathrm{s} 1}=\operatorname{sgn}\left(\Delta P_{\mathrm{s} 1}\right) A_{\mathrm{s} 1}\left(\bar{x}_{\mathrm{s}}\right) \sqrt{\left|\Delta P_{\mathrm{s} 1}\right|} \\
& q_{\mathrm{s} 2}=\operatorname{sgn}\left(\Delta P_{\mathrm{s} 2}\right) A_{\mathrm{s} 2}\left(\bar{x}_{\mathrm{s}}\right) \sqrt{\left|\Delta P_{\mathrm{s} 2}\right|} \\
& q_{1 \mathrm{t}}=\operatorname{sgn}\left(\Delta P_{1 \mathrm{t}}\right) A_{1 \mathrm{t}}\left(\bar{x}_{\mathrm{s}}\right) \sqrt{\left|\Delta P_{1 \mathrm{t}}\right|} \\
& q_{2 \mathrm{t}}=\operatorname{sgn}\left(\Delta P_{2 \mathrm{t}}\right) A_{2 \mathrm{t}}\left(\bar{x}_{\mathrm{s}}\right) \sqrt{\left|\Delta P_{2 \mathrm{t}}\right|}
\end{aligned}
$$

$A_{i j}$ is the pseudo-section of the port $i$ to port $j$ orifice. These pseudo-section functions are implemented with hyperbolic functions. The main reason to use these types of function is the possibility of considering two well-defined asymptotes.

For a symmetrical and centred valve with complete matching of the spool and valve orifices, the number of different pseudo-section functions can be reduced [12]. In this case, the pseudo-sections are related by

$$
A_{\mathrm{s} 1}\left(\bar{x}_{\mathrm{s}}\right)=A_{2 \mathrm{t}}\left(\bar{x}_{\mathrm{s}}\right)=A_{\mathrm{s} 2}\left(-\bar{x}_{\mathrm{s}}\right)=A_{1 \mathrm{t}}\left(-\bar{x}_{\mathrm{s}}\right)
$$

A characteristic of some other valves is that their orifices are symmetrical but not matched, leading to

$$
A_{\mathrm{s} 1}\left(\bar{x}_{\mathrm{s}}\right)=A_{\mathrm{s} 2}\left(-\bar{x}_{\mathrm{s}}\right), \quad A_{1 \mathrm{t}}\left(\bar{x}_{\mathrm{s}}\right)=A_{2 \mathrm{t}}\left(-\bar{x}_{\mathrm{s}}\right)
$$

Using this approach the following functions were used for the pseudo-sections:

$$
\begin{gathered}
A_{\mathrm{s} 2}\left(\bar{x}_{\mathrm{s}}\right)=k_{1} \bar{x}_{\mathrm{s}}+k_{2}+\sqrt{k_{3} \bar{x}_{\mathrm{s}}^{2}+k_{4} \bar{x}_{\mathrm{s}}+k_{5}}, \\
k_{3} \bar{x}_{\mathrm{s}}^{2}+k_{4} \bar{x}_{\mathrm{s}}+k_{5} \geqslant 0, \forall \bar{x}_{\mathrm{s}} \in[-1,1] \\
A_{\mathrm{s} 1}\left(\bar{x}_{\mathrm{s}}\right)=-k_{1} \bar{x}_{\mathrm{s}}+k_{2}+\sqrt{k_{3} \bar{x}_{\mathrm{s}}^{2}-k_{4} \bar{x}_{\mathrm{s}}+k_{5}}, \\
k_{3} \bar{x}_{\mathrm{s}}^{2}-k_{4} \bar{x}_{\mathrm{s}}+k_{5} \geqslant 0, \forall \bar{x}_{\mathrm{s}} \in[-1,1] \\
A_{1 \mathrm{t}}\left(\bar{x}_{\mathrm{s}}\right)=k_{1 t} \bar{x}_{\mathrm{s}}+k_{2 \mathrm{t}}+\sqrt{k_{3 \mathrm{t}} \bar{x}_{\mathrm{s}}^{2}+k_{4 \mathrm{t}} \bar{x}_{\mathrm{s}}+k_{5 \mathrm{t}}}, \\
k_{3 \mathrm{t}} \bar{x}_{\mathrm{s}}^{2}+k_{4 \mathrm{t}} \bar{x}_{\mathrm{s}}+k_{5 \mathrm{t}} \geqslant 0, \forall \bar{x}_{\mathrm{s}} \in[-1,1] \\
A_{2 \mathrm{t}}\left(\bar{x}_{\mathrm{s}}\right)=-k_{1} \bar{x}_{\mathrm{s}}+k_{2 t}+\sqrt{k_{3 \mathrm{t}} \bar{x}_{\mathrm{s}}^{2}-k_{4 \mathrm{t}} \bar{x}_{\mathrm{s}}+k_{5 \mathrm{t}}}, \\
k_{3 \mathrm{t}} \bar{x}_{\mathrm{s}}^{2}-k_{4 \mathrm{t}} \bar{x}_{\mathrm{s}}+k_{5 \mathrm{t}} \geqslant 0, \forall \bar{x}_{\mathrm{s}} \in[-1,1]
\end{gathered}
$$

with $k_{i}, k_{i \mathrm{t}} \in \mathfrak{R}$.

Using the notation in the valve diagrams presented in Figs 3 and 5 , the tank flow $Q_{\mathrm{t}}$, the source flow $Q_{\mathrm{s}}$ and

Proc. Instn Mech. Engrs Vol. 218 Part I: J. Systems and Control Engineering 
the outlet flows $Q_{1}$ and $Q_{2}$ are formulated as follows:

$$
\begin{aligned}
& Q_{\mathrm{s}}=q_{\mathrm{s} 1}+q_{\mathrm{s} 2} \\
& Q_{\mathrm{t}}=q_{1 \mathrm{t}}+q_{2 \mathrm{t}} \\
& Q_{1}=q_{\mathrm{s} 1}-q_{1 \mathrm{t}} \\
& Q_{2}=q_{2 \mathrm{t}}-q_{\mathrm{s} 2}
\end{aligned}
$$

\subsection{Cylinder model}

This section presents the cylinder model including the piston dynamics, the effective bulk modulus and the seal friction. The overall behaviour of the cylinder model is described with a hybrid statechart.

\subsubsection{Piston dynamics}

Considering the schematic diagram presented in Fig. 3, the cylinder dynamics can be defined as

$$
M a_{\mathrm{p}}=P_{1} A_{1}-P_{2} A_{2}-F_{\mathrm{f}}+F_{\mathrm{L}}
$$

where $P_{1}$ and $P_{2}$ are the cylinder chamber pressures, $a_{\mathrm{p}}=\mathrm{d} v_{\mathrm{p}} / \mathrm{d} t$ is the piston acceleration, $v_{\mathrm{p}}$ is the piston velocity, $F_{\mathrm{L}}$ is the total load force, $F_{\mathrm{f}}$ is the frictional force and $M$ is the total mass in motion (load, piston and rod).

The inlet and outlet chamber flows are related to the piston velocity and the compressibility effects. The most important of these effects is the oil compressibility expressed in terms of the bulk modulus. The continuity equation [13], normally taken for a constant oil bulk modulus, can be generalized assuming isothermal conditions (see the Appendix) to use the pressure-dependent effective bulk modulus and expressed as

$$
\sum Q_{\text {in }}-\sum Q_{\text {out }}=\frac{\mathrm{d} V_{0}}{\mathrm{~d} t}+\frac{V_{0}}{\beta(P)} \frac{\mathrm{d} P}{\mathrm{~d} t}
$$

Considering the diagram presented in Fig. 3, the cylinder flowrate can be stated by applying equation (6) to each cylinder chamber:

$$
\begin{aligned}
& Q_{1}=g_{\mathrm{lkc}}\left(P_{1}-P_{2}\right)+A_{1} v_{\mathrm{p}}+\frac{V_{\mathrm{L} 1}+A_{1} x_{\mathrm{p}}}{\beta_{\mathrm{e} 1}\left(P_{1}\right)} \frac{\mathrm{d} P_{1}}{\mathrm{~d} t} \\
& Q_{2}=-g_{\mathrm{lkc}}\left(P_{1}-P_{2}\right)+A_{2} v_{\mathrm{p}}-\frac{V_{\mathrm{L} 2}+A_{2}\left(L-x_{\mathrm{p}}\right)}{\beta_{\mathrm{e} 2}\left(P_{2}\right)} \frac{\mathrm{d} P_{2}}{\mathrm{~d} t}
\end{aligned}
$$

It should be noted that the volume in chamber 1 is $V_{01}=V_{\mathrm{L} 1}+A_{1} x_{\mathrm{p}}$ and in chamber 2 this volume is $V_{02}=V_{\mathrm{L} 2}+A_{2}\left(L-x_{\mathrm{p}}\right) . V_{\mathrm{L} 1}$ and $V_{\mathrm{L} 2}$ represent the line volumes plus the cylinder dead volumes and respective valve chamber volumes $\beta_{\mathrm{e} 1}$ and $\beta_{\mathrm{e} 2}$ represent the compressibilities of the fluid, pipes and cylinder walls of chambers 1 and 2 respectively; i.e. they are the effective bulk moduli. The external leakages are considered to be negligible and the cylinder internal leakage flowrate, which is assumed to be laminar, is given by $q_{1 \mathrm{kc}}=g_{1 \mathrm{kc}}\left(P_{1}-P_{2}\right)$, where $g_{\mathrm{lkc}}$ is the leakage conductance. The piston velocity is $v_{\mathrm{p}}=\mathrm{d} x_{\mathrm{p}} / \mathrm{d} t$, where $x_{\mathrm{p}}$ is the piston position.

\subsubsection{Effective bulk modulus}

Most of the times, the effective bulk modulus is made constant in simulations. However, when hydraulic systems have to run over a wide pressure range, a value for $\beta_{\mathrm{e}}$ that is pressure dependent, is needed to achieve satisfactory simulation results. To model the effective bulk modulus, a pressure-dependent model proposed in reference $[\mathbf{1 5}]$ is used. The model assumes that the air volume mixed in the oil is negligible, that the oil temperature and pumping conditions are constant and that rigid pipes are used. The effective bulk modulus can then be stated for both cylinder chambers:

$$
\beta_{\mathrm{e} 1}=\frac{10^{5}+P_{1}}{B P_{1}+C}, \quad \beta_{\mathrm{e} 2}=\frac{10^{5}+P_{2}}{B P_{2}+C}
$$

where $B\left[P_{a}^{-1}\right]$ and $C$ are constants related to the oil characteristics.

\subsubsection{Friction model}

The friction phenomena are highly non-linear and can cause considerable steady state errors and low performance in hydraulically controlled systems. Friction is the reaction tangential force between two sliding surfaces. In a hydraulic cylinder, most of the friction effects are generated in the contact of the seals with the cylinder body. Different friction patterns occur for different velocities and motion states. For small velocities, the hydraulic fluid acts as a superficial layer and the shear forces determine the friction. For high velocities and low pressures, a permanent fluid layer is developed and the friction is related to hydrodynamic effects. The friction then depends on the fluid viscosity characteristics and also on the distribution of velocities along the fluid layer. In the past, several methods to model the friction were proposed. Most of these are static models and have different complexities [16]. However, in recent years, interest on dynamic models for friction grew, especially in the analysis of high-accuracy servo-systems and in the development of friction compensators for control purposes [17-20].

To model the friction between the seals and cylinder body, the LuGre dynamic model was used. The LuGre friction model [21] can describe complex friction behaviour as the pre-sliding displacement, the stick-slip motion, the Stribeck effects and frictional lag. The stick-slip motion consists of a limit cycle oscillation at low velocities that occurs because the frictional force is larger at rest than during motion. The pre-sliding displacement shows that the friction behaves like a spring when the applied resulting force is lower than the static breakaway force. The frictional lag means that there is hysteresis in the 
frictional force versus velocity curves. The LuGre model is based on the interpretation of the friction as the force generated by a set of bristles belonging to the two surfaces in contact. The elastic deformation, corresponding to small displacements, is described by having spring behaviour given by the bristles. For large displacements, the plastic deformation is captured by the movement of the bristle's connections; i.e. some connections are broken and others established. The model formulation is based on the average behaviour of the bristles in contact. In a hydraulic cylinder the seal can be considered as the bristle of the LuGre model. For a steady state velocity the seal deflection (state variable $z$ ) is constant.

The seal deformation (Fig. 6) is represented by a state variable $z$ whose dynamics are given by

$$
\frac{\mathrm{d} z}{\mathrm{~d} t}=v_{\mathrm{p}}-\frac{\left|v_{\mathrm{p}}\right|}{g\left(v_{\mathrm{p}}\right)} z
$$

where $v_{\mathrm{p}}$ is the piston velocity and $g(v)$ is given by

$$
g\left(v_{\mathrm{p}}\right)=\frac{1}{\sigma_{0}}\left(F_{\mathrm{CO}}+\left(F_{\mathrm{S}}-F_{\mathrm{CO}}\right) \mathrm{e}^{-\left(v_{\mathrm{p}} / v_{\mathrm{S}}\right)^{2}}\right)
$$

$F_{\mathrm{CO}}$ is the Coulomb frictional force, $F_{\mathrm{S}}$ is the Stribeck force, $v_{\mathrm{S}}$ is the Stribeck velocity and $\sigma_{0}$ is the seal stiffness.

The frictional force is generated due to the bending of the seal that acts as a spring and damper, being proportional to the seal deflection, its rate of change and piston velocity. The frictional force is then given by

$$
F_{\mathrm{f}}=\sigma_{0} z+\sigma_{1} \frac{\mathrm{d} z}{\mathrm{~d} t}+K_{\mathrm{v}} v_{\mathrm{p}}
$$

where $\sigma_{1}$ is the damping factor of the seal motion and $K_{\mathrm{v}}$ is the viscous friction coefficient.

For small displacements the model has a behaviour identical with a spring with a stiffness coefficient $\sigma_{0}$ and a damping factor $\sigma_{1}$. The maximum seal deformation occurs in the pre-sliding phase (velocity is zero) and is given by $F_{\mathrm{S}} / \sigma_{0}$.

\subsubsection{Cylinder model's behaviour using a statechart}

In real-time simulation all modes of operation can be tested including failure modes. For that reason the piston displacement has to be limited between 0 and $L$.
Accurate modelling of the behaviour, when the piston reaches the extremes, implies the use of a mechanical impact with fast dynamics that increases the model's stiffness and makes impossible the real-time simulation in low-cost hardware. In some situations the impact dynamics can be handled with a simple model to limit the cylinder work length. The way that the model should be implemented can be well described through a hybrid statechart.

Using the hybrid statecharts formalism the cylinder behaviour can be organized in three parallel 'tasks'. These correspond to the high-level description of the AND state Cylinder with three orthogonal states Piston, BulkModulus and SealFriction (Fig. 7).

These states can be refined through state decomposition. For example, the state Piston, used to describe the piston and chamber pressure dynamics, can be a compound OR state with substates StopLeft, Normal and StopRight (Fig. 8).

The state decomposition process can proceed until a low-level description is achieved. For example the StopLeft and StopRight states are then decomposed in order to describe the cylinder behaviour when it is stopped at the extremes. The main functionality to handle the hard cylinder stops is the restarting of the state variable $v_{\mathrm{p}}$ when the piston reaches one of the limits $\left(x_{\mathrm{p}}<0\right.$ or $\left.x_{\mathrm{p}}>L\right)$. When such an event occurs, the cylinder 'enters' a stop state, and its acceleration is set to zero. To 'leave' the stop state the applied force (hydraulic force, load and friction) must invert its signal. During activities define the continuous state behaviour. The Normal state specifies that the piston acceleration $a_{\mathrm{p}}$ is calculated using the Newton second law, where $f$ is the resulting force applied to the piston. When the StopLeft or StopRight state is entered, the action associated with the state entrance resets the piston velocity state variable. At the same time, and considering that the piston enters in the StopRight state, the default state PosForceR is also entered and becomes active, which makes active its continuous activity, with the piston acceleration now set to zero. In the meantime, if the resulting force, evaluated in the During activity of the Piston state, inverts its signal, the piston acceleration is again calculated with the Newton law, because the NegForceR state becomes active. It should be noted that all the During activities

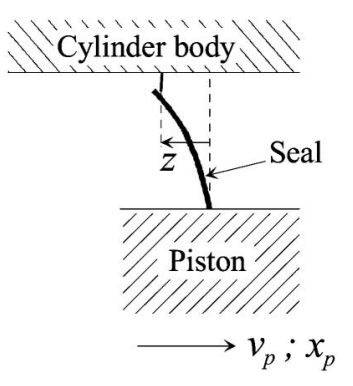

Fig. 6 Deformation of the cylinder seal

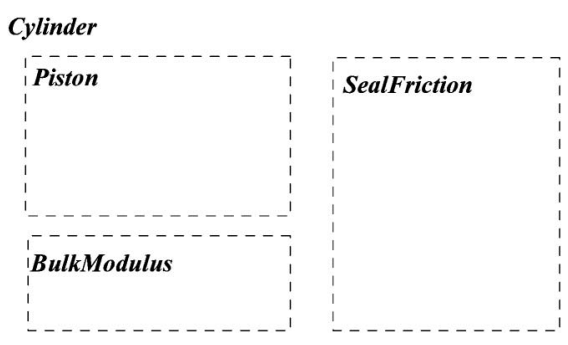

Fig. 7 High-level cylinder statechart

Proc. Instn Mech. Engrs Vol. 218 Part I: J. Systems and Control Engineering 


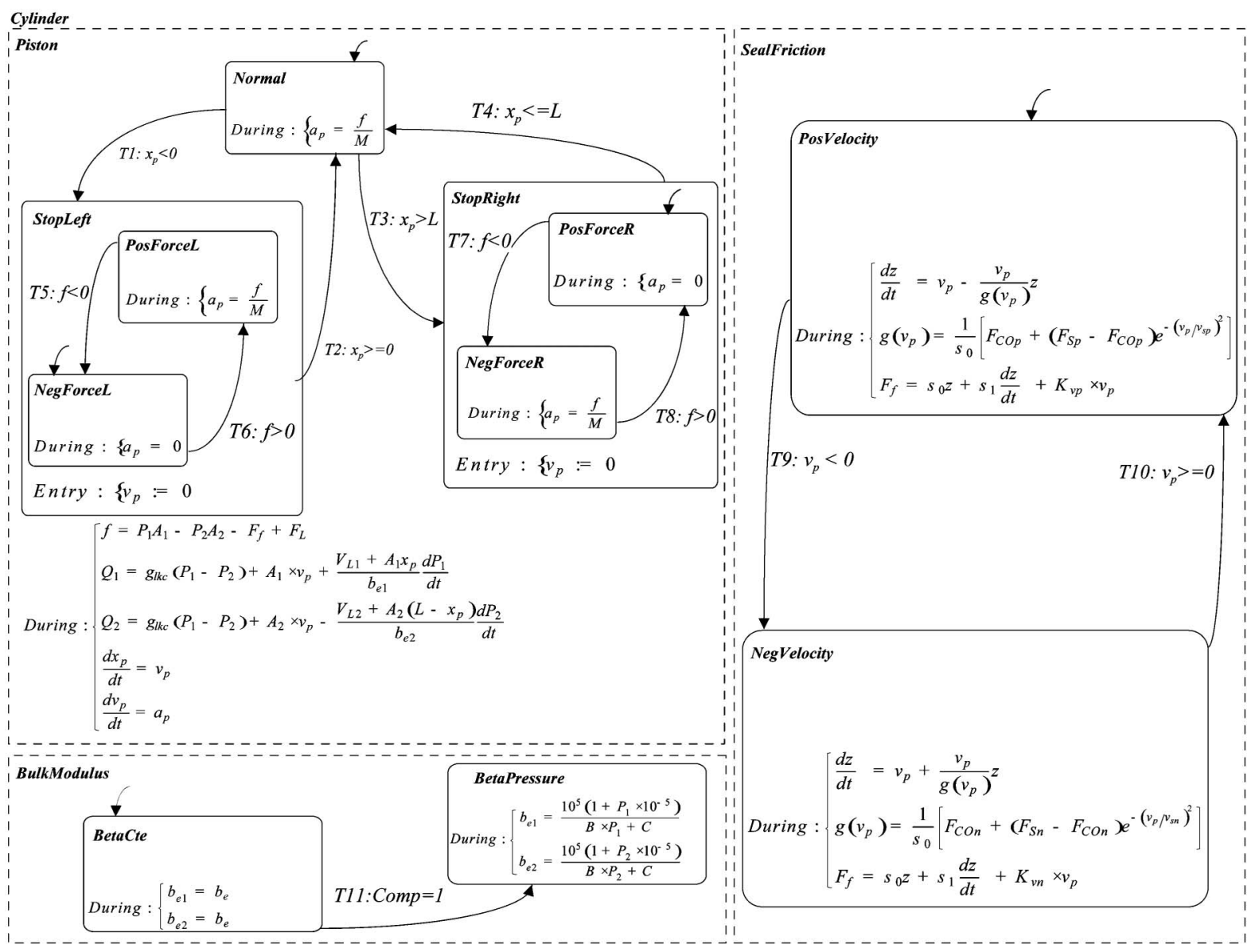

Fig. 8 Low-level cylinder statechart with all the continuous activities and event actions defined

of the active states constitute the DAEs that have to be solved to simulate the system behaviour in the present continuous mode. With the broadcast communication mechanism, all the actions and During activities are 'sensed' at the same time; i.e., in this case, all the variables are 'visible' in all the states.

It is interesting to point out that statecharts can be used to handle models with different complexities, appropriated to different types of simulation. For instance, the substates BetaCte and BetaPressure can define, in their During activities, different equations for the effective bulk moduli of the cylinder chambers. The model user can then select the bulk modulus equations to use in a certain simulation by just changing the Comp variable to the desired complexity level. Thus, at the beginning of the simulation process, the transition $T 11$ is taken, or not, defining in that way the bulk modulus equations to be used.

\section{CONCLUSIONS}

The present work proposes two complementary approaches for the modelling of hydraulic systems to perform HILS experiments using low-cost real-time hardware. The main goal was to develop not too complex and not too stiff hydraulic component models for real-time simulation.

The first step is a reduction in the model's stiffness by considering some fast dynamics, such as the piston impact on the cylinder extremes, as instantaneous changes. This results in models with multi-continuous modes, interspersed with instantaneous transitions that can be successfully represented by the statecharts formalism with hybrid features. The overall cylinder behaviour is described by a hybrid statechart that proves suitable to represent graphically the variable equation structure and also different complexities of the cylinder model.

The other approach is to reduce the overall model complexity by the use of semi-empirical component models. A model of a hydraulic system composed of a highperformance proportional valve and a hydraulic cylinder was fully developed. A semi-empirical valve model that describes the volumetric flowrates of proportional valves with different orifice sections was proposed. The section orifices follow hyperbolic functions obtained with the spool position as input. The volumetric flowrate through the valve orifices is considered to be always turbulent, being the laminar flow implicitly modelled in the section functions. The use of a dynamic model to describe the cylinder seal friction is also proposed.

I03404 (C) IMechE 2004 


\section{Acknowledgement}

This work was funded by FCT under the programme POCTI.

\section{REFERENCES}

1 Maclay, D. Simulation gets into the loop. Instn Electl Engrs Rev, May 1997, 109-112.

2 Mosterman, P. J. and Biswas, G. Building hybrid automata of complex physical systems for real-time applications. In Proceedings of the Conference on Decision and Control, Phoenix, Arizona, 1999, pp. 3514-3519.

3 Mosterman, P. J. and Biswas, G. Modeling discontinuous behavior with hybrid bond graphs. In Proceedings of the 1995 International Conference on Qualitative Reasoning, Amsterdam, The Netherlands, 1995, pp. 139-147.

4 Alur, R., Courcoubetis, C., Henzinger, T. A. and Ho, P. Hybrid automata: an algorithmic approach to the specification and verification of hybrid systems. In Hybrid Systems I, Lecture Notes in Computer Science, Vol. 736, 1993, pp. 209-229 (Springer-Verlag, Berlin, Germany).

5 Pettersson, S. and Lennartson, B. Hybrid modelling focused on hybrid Petri nets. In Proceedings of the 2nd European Workshop on Hybrid Systems, Grenoble, France, 1995, pp. 303-309.

6 Harel, D. Statecharts: a visual formalism for complex systems. Sci. Computer Programming, 1987, 8, 231-274.

7 Kesten, Y. and Pnueli, A. Timed and hybrid statecharts and their textual representation. In Formal Techniques in RealTime and Fault-Tolerant Systems, Lecture Notes in Computer Science, Vol. 57 (Ed. J. Vytopil), 1992, pp. 591-619 (Springer-Verlag, Berlin, Germany).

8 Ferreira, J. A., Estima de Oliveira, J. P. and Costa, V. A. Modeling of hydraulic systems for hardware-in-the-loop simulation: a methodology proposal. In Proceedings of the 1999 ASME International Mechanical Engineering Congress and Exposition-Fluid Power System and Technology, Nashville, Tennessee, 1999, FPST-Vol. 6, pp. 33-39.

9 Beeck, M. A comparation of statecharts variants. In Lecture Notes in Computer Science, Vol. 863, 1994, (SpringerVerlag, Berlin).

10 Beater, P. Object oriented modelling and simulation of hydraulic drives. Simulation News Europe, March 1998.

11 Quintas, M. R. Contribution à la modélisation et à la command robust de sistèmes électrohydrauliques. Docteur Thèse, L'Institute National des Sciences Appliquees de Lyon, France, 1999.

12 Ferreira, J. A., Gomes de Almeida, F. and Quintas, M. R. Semi-empirical model for a hydraulic servo-solenoid valve. Proc. Instn Mech. Engrs, Part I: J. Systems and Control Engineering, 2002, 216(I3), 237-248.

13 Merrit, H. E. Hydraulic Control Systems, 1967 (John Wiley, New York)

14 Watton, J. Fluid Power Systems-Modeling Simulation, Analog and Microcomputer Control, 1989 (Prentice-Hall, UK).
15 Yu, J., Chen, Z. and Lu, Y. The variation of oil effective bulk modulus with pressure in hydraulic systems. Trans. ASME, J. Dynamic Systems, Measmt Control, March 1994, 116, 146-149.

16 Armstrong, B., Dupont, P. and Canudas de Wit, C. A survey of models, analysis tools and compensation methods for the control of machines with friction. Automatica, 1994, 30(7), 1083-1138.

17 Southward, S., Radcliffe, C. and MacCluer, C. Robust Nonlinear Stick-Slic Friction Compensation. Trans. ASME, J. Dynamic Systems, Measmt Control, December 1991, 113, 639-645.

18 Lischinsky, P., Canudas de Wit, C. and Morel, G. Friction compensation for an Industrial hydraulic robot. IEEE Control Systems Mag., 1999, 19(1), 25-32.

19 Swevers, J., Al-Bender, F., Ganseman, C. G. and Prajogo, T. An integrated friction model structure with improved presliding behaviour for accurate friction compensation. IEEE Trans. Autom. Control, April 2000, 45(4), 675-686.

20 Tan, K. K., Lee, T. H., Huang, S. N. and Jiang, X. Friction modeling and adaptive compensation using a relay feedback approach. IEEE Trans. Ind. Electron., February 2001, 48(1), 169-176.

21 Canudas de Wit, C., Olsson, H., Aström, K. J. and Lischinsky, P. A new model for control of systems with friction. IEEE Trans. Autom. Control, March 1995, 40(3), 419-425.

\section{APPENDIX}

Considering a control volume with a volume $V_{0}$, a stored mass of a fluid inside, $m$, with a mass density $\rho$, the mass balance equation specifies that the rate at which the mass is stored must equal the incoming weight flowrate minus the outgoing weight flowrate [13]:

$$
\sum W_{\text {in }}-\sum W_{\text {out }}=g \frac{\mathrm{d} m}{\mathrm{~d} t}=g \frac{\mathrm{d}\left(\rho V_{0}\right)}{\mathrm{d} t}
$$

As the oil density is a function of temperature and pressure, $\rho=\rho(T, P)$, the following can then be stated:

$$
\mathrm{d} \rho=\left(\frac{\partial \rho}{\partial T}\right)_{P} \mathrm{~d} T+\left(\frac{\partial \rho}{\partial P}\right)_{T} \mathrm{~d} P
$$

Assuming isothermal conditions the state equation for the oil can be simplified to

$$
\mathrm{d} \rho=\left(\frac{\partial \rho}{\partial P}\right)_{T} \mathrm{~d} P
$$

The isothermal tangent bulk modulus, which is pressure dependent, is defined as

$$
\beta(P)=\rho\left(\frac{\partial P}{\partial \rho}\right)_{T}
$$


Then, using $\rho / \beta(P)=(\partial \rho / \partial P)_{T}$ in equation (15) gives

$$
\frac{1}{\rho} \mathrm{d} \rho=\frac{1}{\beta(P)} \mathrm{d} P
$$

If $\rho_{0}$ is assumed to be the oil density at pressure $P_{0}$, the following expression is obtained for $\rho$ at pressure $P$ :

$$
\rho=\rho_{0} \mathrm{e}^{\int_{P_{0}}^{P}[1 / \beta(P)] \mathrm{d} P}
$$

Considering that the incoming oil and outgoing oil are at the same pressure as the chamber oil, equation (13) can be simplified to relate the flowrates:

$$
\sum Q_{\text {in }}-\sum Q_{\text {out }}=\frac{\mathrm{d} V_{0}}{\mathrm{~d} t}+\frac{V_{0}}{\rho} \frac{\mathrm{d} \rho}{\mathrm{d} t}
$$

where $Q$ is the volumetric flowrate $(W=g \rho Q)$.
As $\mathrm{d} \rho / \mathrm{d} t=(\mathrm{d} \rho / \mathrm{d} P)(\mathrm{d} P / \mathrm{d} t),(\mathrm{d} \rho / \mathrm{d} P)$ can be calculated for $\rho=\rho_{0} \mathrm{e}^{\int_{P_{0}}^{P}[1 / \beta(P)] \mathrm{d} P \text {, }}$

$$
\frac{\mathrm{d} \rho}{\mathrm{d} P}=\rho_{0} \frac{\mathrm{d}\left(\int_{P_{0}}^{P}[1 / \beta(P)] \mathrm{d} P\right)}{\mathrm{d} P}
$$

Then, from equation (17),

$$
\frac{\mathrm{d} \rho}{\mathrm{d} P}=\rho \frac{1}{\beta(P)}
$$

and equation (19) can be written as

$$
\sum Q_{\text {in }}-\sum Q_{\text {out }}=\frac{\mathrm{d} V_{0}}{\mathrm{~d} t}+\frac{V_{0}}{\beta(P)} \frac{\mathrm{d} P}{\mathrm{~d} t}
$$

